MATHEMATICS OF COMPUTATION

Volume 76, Number 259, July 2007, Pages 1469-1481

S 0025-5718(07)01931-X

Article electronically published on January 24, 2007

\title{
ERROR BOUNDS ON COMPLEX FLOATING-POINT MULTIPLICATION
}

\author{
RICHARD BRENT, COLIN PERCIVAL, AND PAUL ZIMMERMANN
}

In memory of Erin Brent (1947-2005)

\begin{abstract}
Given floating-point arithmetic with $t$-digit base- $\beta$ significands in which all arithmetic operations are performed as if calculated to infinite precision and rounded to a nearest representable value, we prove that the product of complex values $z_{0}$ and $z_{1}$ can be computed with maximum absolute error $\left|z_{0} \| z_{1}\right| \frac{1}{2} \beta^{1-t} \sqrt{5}$. In particular, this provides relative error bounds of $2^{-24} \sqrt{5}$ and $2^{-53} \sqrt{5}$ for IEEE 754 single and double precision arithmetic respectively, provided that overflow, underflow, and denormals do not occur.

We also provide the numerical worst cases for IEEE 754 single and double precision arithmetic.
\end{abstract}

\section{INTRODUCTION}

In an earlier paper 2, the second author made the claim that the maximum relative error which can occur when computing the product $z_{0} z_{1}$ of two complex values using floating-point arithmetic is $\epsilon \sqrt{5}$, where $\epsilon$ is the maximum relative error which can result from rounded floating-point addition, subtraction, or multiplication. While reviewing that paper a few years later, the other two authors noted that the proof given was incorrect, although the result claimed was true.

Since the bound of $\epsilon \sqrt{8}$ which is commonly used [1] is suboptimal, we present here a corrected proof of the tighter bound. Interestingly, by explicitly finding worst-case inputs, we can demonstrate that our error bound is effectively optimal.

Throughout this paper, we concern ourselves with floating-point arithmetic with $t$-digit base- $\beta$ significands, denote by $\operatorname{ulp}(x)$ for $x \neq 0$ the (unique) power of $\beta$ such that $\beta^{t-1} \leq|x| / \operatorname{ulp}(x)<\beta^{t}$, and write $\epsilon=\frac{1}{2} \operatorname{ulp}(1)=\frac{1}{2} \beta^{1-t}$; we also define $\operatorname{ulp}(0)=0$. The notations $x \oplus y, x \ominus y$, and $x \otimes y$ represent rounded-to-nearest floating-point addition, subtraction, and multiplication of the values $x$ and $y$.

\section{AN ERROR BOUND}

Theorem 1. Let $z_{0}=a_{0}+b_{0} i$ and $z_{1}=a_{1}+b_{1} i$, with $a_{0}, b_{0}, a_{1}, b_{1}$ floatingpoint values with $t$-digit base- $\beta$ significands, and let $z_{2}=\left(\left(a_{0} \otimes a_{1}\right) \ominus\left(b_{0} \otimes b_{1}\right)\right)+$ $\left(\left(a_{0} \otimes b_{1}\right) \oplus\left(b_{0} \otimes a_{1}\right)\right) i$ be computed. Providing that no overflow or underflow occurs, no denormal values are produced, arithmetic results are correctly rounded to

Received by the editor November 21, 2005 and, in revised form, February 21, 2006.

2000 Mathematics Subject Classification. Primary 65G50.

(C)2007 American Mathematical Society Reverts to public domain 28 years from publication 
a nearest representable value, $z_{0} z_{1} \neq 0$, and $\epsilon \leq 2^{-5}$, the relative error

$$
\left|z_{2}\left(z_{0} z_{1}\right)^{-1}-1\right|
$$

is less than $\epsilon \sqrt{5}=\frac{1}{2} \beta^{1-t} \sqrt{5}$.

Proof. Let $a_{0}, b_{0}, a_{1}$, and $b_{1}$ be chosen such that the relative error is maximized. By multiplying $z_{0}$ and $z_{1}$ by powers of $i$ and/or taking complex conjugates, we can assume, without loss of generality, that

$$
\begin{aligned}
0 & \leq a_{0}, b_{0}, a_{1}, b_{1}, \\
b_{0} b_{1} & \leq a_{0} a_{1},
\end{aligned}
$$

and given our assumptions that overflow, underflow, and denormals do not occur, and that rounding is performed to a nearest representable value, we can conclude that for any $x$ occurring in the computation, the error introduced when rounding $x$ is at most $\frac{1}{2} \operatorname{ulp}(x)$ and is strictly less than $\epsilon \cdot x$.

We note that the error $\left|\Im\left(z_{2}-z_{0} z_{1}\right)\right|$ in the imaginary part of $z_{2}$ is bounded as follows:

$$
\begin{aligned}
\left|\Im\left(z_{2}-z_{0} z_{1}\right)\right|= & \left|\left(\left(a_{0} \otimes b_{1}\right) \oplus\left(b_{0} \otimes a_{1}\right)\right)-\left(a_{0} b_{1}+b_{0} a_{1}\right)\right| \\
\leq & \left|a_{0} \otimes b_{1}-a_{0} b_{1}\right|+\left|b_{0} \otimes a_{1}-b_{0} a_{1}\right| \\
& \quad+\left|\left(\left(a_{0} \otimes b_{1}\right) \oplus\left(b_{0} \otimes a_{1}\right)\right)-\left(a_{0} \otimes b_{1}+b_{0} \otimes a_{1}\right)\right| .
\end{aligned}
$$

Now we consider two cases:

Case I1. $\operatorname{ulp}\left(a_{0} b_{1}+b_{0} a_{1}\right)<\operatorname{ulp}\left(a_{0} \otimes b_{1}+b_{0} \otimes a_{1}\right)$.

Using first the definition of ulp and second the assumption above, we must have

$$
a_{0} b_{1}+b_{0} a_{1}<\beta^{t} \mathrm{ulp}\left(a_{0} b_{1}+b_{0} a_{1}\right) \leq a_{0} \otimes b_{1}+b_{0} \otimes a_{1}
$$

and therefore

$$
\begin{aligned}
\left|\left(a_{0} \otimes b_{1}+b_{0} \otimes a_{1}\right)-\beta^{t} \mathrm{ulp}\left(a_{0} b_{1}+b_{0} a_{1}\right)\right| & <\left(a_{0} \otimes b_{1}+b_{0} \otimes a_{1}\right)-\left(a_{0} b_{1}+b_{0} a_{1}\right) \\
& \leq\left|a_{0} \otimes b_{1}-a_{0} b_{1}\right|+\left|b_{0} \otimes a_{1}-b_{0} a_{1}\right| \\
& \leq \epsilon \cdot\left(a_{0} b_{1}+b_{0} a_{1}\right) .
\end{aligned}
$$

However, $\beta^{t} \operatorname{ulp}\left(a_{0} b_{1}+b_{0} a_{1}\right)$ is a representable floating-point value; so given our assumption that rounding is performed to a nearest representable value, we must now have

$$
\left|\left(\left(a_{0} \otimes b_{1}\right) \oplus\left(b_{0} \otimes a_{1}\right)\right)-\left(a_{0} \otimes b_{1}+b_{0} \otimes a_{1}\right)\right|<\epsilon \cdot\left(a_{0} b_{1}+b_{0} a_{1}\right) .
$$

Case I2. $\operatorname{ulp}\left(a_{0} \otimes b_{1}+b_{0} \otimes a_{1}\right) \leq u \operatorname{up}\left(a_{0} b_{1}+b_{0} a_{1}\right)$.

From our assumption that the results of arithmetic operations are correctly rounded, we obtain

$$
\begin{aligned}
\left|\left(\left(a_{0} \otimes b_{1}\right) \oplus\left(b_{0} \otimes a_{1}\right)\right)-\left(a_{0} \otimes b_{1}+b_{0} \otimes a_{1}\right)\right| & \leq \frac{1}{2} \mathrm{ulp}\left(a_{0} \otimes b_{1}+b_{0} \otimes a_{1}\right) \\
& \leq \frac{1}{2} \mathrm{ulp}\left(a_{0} b_{1}+b_{0} a_{1}\right) \\
& \leq \epsilon \cdot\left(a_{0} b_{1}+b_{0} a_{1}\right) .
\end{aligned}
$$


Combining these two cases with the earlier-stated bound, we obtain

$$
\begin{aligned}
&\left|\Im\left(z_{2}-z_{0} z_{1}\right)\right| \leq\left|a_{0} \otimes b_{1}-a_{0} b_{1}\right|+\left|b_{0} \otimes a_{1}-b_{0} a_{1}\right| \\
&+\left|\left(\left(a_{0} \otimes b_{1}\right) \oplus\left(b_{0} \otimes a_{1}\right)\right)-\left(a_{0} \otimes b_{1}+b_{0} \otimes a_{1}\right)\right| \\
&<\epsilon \cdot\left(a_{0} b_{1}\right)+\epsilon \cdot\left(b_{0} a_{1}\right)+\epsilon \cdot\left(a_{0} b_{1}+b_{0} a_{1}\right) \\
&=\epsilon \cdot\left(2 a_{0} b_{1}+2 b_{0} a_{1}\right) .
\end{aligned}
$$

Now that we have a bound on the imaginary part of the error, we turn our attention to the real part, and consider the following four cases (where the examples given apply to $\beta=2$ ):

$$
\begin{array}{ll}
\mathrm{ulp}\left(b_{0} b_{1}\right) \leq \mathrm{ulp}\left(a_{0} a_{1}\right) \leq \mathrm{ulp}\left(a_{0} \otimes a_{1}-b_{0} \otimes b_{1}\right), & \text { e.g., } z_{0}=z_{1}=0.8+0.1 i, \\
\mathrm{ulp}\left(b_{0} b_{1}\right)<\mathrm{ulp}\left(a_{0} \otimes a_{1}-b_{0} \otimes b_{1}\right)<\operatorname{ulp}\left(a_{0} a_{1}\right), & \text { e.g., } z_{0}=z_{1}=0.8+0.4 i, \\
\mathrm{ulp}\left(a_{0} \otimes a_{1}-b_{0} \otimes b_{1}\right) \leq \mathrm{ulp}\left(b_{0} b_{1}\right)<\operatorname{ulp}\left(a_{0} a_{1}\right), & \text { e.g., } z_{0}=z_{1}=0.8+0.7 i, \\
\mathrm{ulp}\left(a_{0} \otimes a_{1}-b_{0} \otimes b_{1}\right)<\mathrm{ulp}\left(b_{0} b_{1}\right)=\mathrm{ulp}\left(a_{0} a_{1}\right), & \text { e.g., } z_{0}=z_{1}=0.8+0.8 i .
\end{array}
$$

Since we have assumed that $b_{0} b_{1} \leq a_{0} a_{1}$, we know that $\operatorname{ulp}\left(b_{0} b_{1}\right) \leq \operatorname{ulp}\left(a_{0} a_{1}\right)$, and thus these four cases cover all possible inputs. Consequently, it suffices to prove the required bound for each of these four cases.

Case R1. ulp $\left(b_{0} b_{1}\right) \leq \mathrm{ulp}\left(a_{0} a_{1}\right) \leq \mathrm{ulp}\left(a_{0} \otimes a_{1}-b_{0} \otimes b_{1}\right)$.

Note that the right inequality can only be strict if $a_{0} \otimes a_{1}$ rounds up to a power of $\beta$ and $b_{0} b_{1}=0$.

We observe that

$$
a_{0} \otimes a_{1}-b_{0} \otimes b_{1}<a_{0} a_{1}-b_{0} b_{1}+\epsilon \cdot\left(a_{0} a_{1}+b_{0} b_{1}\right)
$$

and bound the real part of the complex error as follows:

$$
\begin{aligned}
\left|\Re\left(z_{2}-z_{0} z_{1}\right)\right| \leq & \left|a_{0} \otimes a_{1}-a_{0} a_{1}\right|+\left|b_{0} \otimes b_{1}-b_{0} b_{1}\right| \\
& +\left|\left(\left(a_{0} \otimes a_{1}\right) \ominus\left(b_{0} \otimes b_{1}\right)\right)-\left(a_{0} \otimes a_{1}-b_{0} \otimes b_{1}\right)\right| \\
\leq & \frac{1}{2} \mathrm{ulp}\left(a_{0} a_{1}\right)+\frac{1}{2} \mathrm{ulp}\left(b_{0} b_{1}\right)+\frac{1}{2} \mathrm{ulp}\left(a_{0} \otimes a_{1}-b_{0} \otimes b_{1}\right) \\
\leq & \frac{1}{2} \mathrm{ulp}\left(a_{0} \otimes a_{1}-b_{0} \otimes b_{1}\right)+\frac{1}{2} \mathrm{ulp}\left(b_{0} b_{1}\right)+\frac{1}{2} \mathrm{ulp}\left(a_{0} \otimes a_{1}-b_{0} \otimes b_{1}\right) \\
< & 2 \epsilon \cdot\left(a_{0} \otimes a_{1}-b_{0} \otimes b_{1}\right)+\epsilon \cdot\left(b_{0} b_{1}\right) \\
< & <\cdot\left(2 a_{0} a_{1}-b_{0} b_{1}\right)+\epsilon^{2} \cdot\left(2 a_{0} a_{1}+2 b_{0} b_{1}\right) .
\end{aligned}
$$

Applying the triangle inequality, we now observe that

$$
\begin{aligned}
\left|z_{2}-z_{0} z_{1}\right| & =\sqrt{\Re\left(z_{2}-z_{0} z_{1}\right)^{2}+\Im\left(z_{2}-z_{0} z_{1}\right)^{2}} \\
& <\epsilon \sqrt{\left(2 a_{0} a_{1}-b_{0} b_{1}\right)^{2}+\left(2 a_{0} b_{1}+2 b_{0} a_{1}\right)^{2}}+\epsilon^{2} \cdot\left(2 a_{0} a_{1}+2 b_{0} b_{1}\right) \\
& \leq \epsilon \sqrt{\frac{32}{7}\left|z_{0} z_{1}\right|^{2}-\frac{4}{7}\left(a_{0} b_{1}-b_{0} a_{1}\right)^{2}-\frac{1}{7}\left(2 a_{0} a_{1}-5 b_{0} b_{1}\right)^{2}}+2 \epsilon^{2}\left|z_{0} z_{1}\right| \\
& \leq \epsilon(\sqrt{32 / 7}+2 \epsilon)\left|z_{0} z_{1}\right|<\epsilon \sqrt{5}\left|z_{0} z_{1}\right|
\end{aligned}
$$

as required. 
Case R2. ulp $\left(b_{0} b_{1}\right)<\operatorname{ulp}\left(a_{0} \otimes a_{1}-b_{0} \otimes b_{1}\right)<\operatorname{ulp}\left(a_{0} a_{1}\right)$.

Noting that $\operatorname{ulp}(x)<\operatorname{ulp}(y)$ implies $\operatorname{ulp}(x) \leq \beta^{-1} \operatorname{ulp}(y) \leq \frac{1}{2} \mathrm{ulp}(y)$, we obtain

$$
\begin{aligned}
\left|\Re\left(z_{2}-z_{0} z_{1}\right)\right| & \leq \frac{1}{2} \mathrm{ulp}\left(a_{0} a_{1}\right)+\frac{1}{2} \mathrm{ulp}\left(b_{0} b_{1}\right)+\frac{1}{2} \mathrm{ulp}\left(a_{0} \otimes a_{1}-b_{0} \otimes b_{1}\right) \\
& \leq \frac{7}{8} \mathrm{ulp}\left(a_{0} a_{1}\right) \\
& \leq \epsilon \cdot\left(\frac{7}{4} a_{0} a_{1}\right)
\end{aligned}
$$

and therefore

$$
\begin{aligned}
\left|z_{2}-z_{0} z_{1}\right| & =\sqrt{\Re\left(z_{2}-z_{0} z_{1}\right)^{2}+\Im\left(z_{2}-z_{0} z_{1}\right)^{2}} \\
& <\epsilon \sqrt{\left(\frac{7}{4} a_{0} a_{1}\right)^{2}+\left(2 a_{0} b_{1}+2 b_{0} a_{1}\right)^{2}} \\
& =\epsilon \sqrt{\frac{1024}{207}\left|z_{0} z_{1}\right|^{2}-\frac{196}{207}\left(a_{0} b_{1}-b_{0} a_{1}\right)^{2}-\frac{1}{3312}\left(79 a_{0} a_{1}-128 b_{0} b_{1}\right)^{2}} \\
& \leq \epsilon \sqrt{1024 / 207}\left|z_{0} z_{1}\right|<\epsilon \sqrt{5}\left|z_{0} z_{1}\right|
\end{aligned}
$$

as required.

Case R3. ulp $\left(a_{0} \otimes a_{1}-b_{0} \otimes b_{1}\right) \leq \operatorname{ulp}\left(b_{0} b_{1}\right)<\operatorname{ulp}\left(a_{0} a_{1}\right)$.

In this case, there is no rounding error introduced in computing the difference between $a_{0} \otimes a_{1}$ and $b_{0} \otimes b_{1}$ since $\operatorname{ulp}\left(a_{0} \otimes a_{1}-b_{0} \otimes b_{1}\right) \leq \mathrm{ulp}\left(b_{0} b_{1}\right) \leq \mathrm{ulp}\left(b_{0} \otimes b_{1}\right)$ and $\operatorname{ulp}\left(a_{0} \otimes a_{1}-b_{0} \otimes b_{1}\right)<\operatorname{ulp}\left(a_{0} a_{1}\right) \leq \operatorname{ulp}\left(a_{0} \otimes a_{1}\right)$. Also,

$$
\mathrm{ulp}\left(b_{0} b_{1}\right) \leq \frac{1}{\beta} \mathrm{ulp}\left(a_{0} a_{1}\right) \leq \frac{1}{2} \mathrm{ulp}\left(a_{0} a_{1}\right)
$$

so we have

$$
\begin{aligned}
\left|\Re\left(z_{2}-z_{0} z_{1}\right)\right| & \leq \frac{1}{2} \mathrm{ulp}\left(a_{0} a_{1}\right)+\frac{1}{2} \mathrm{ulp}\left(b_{0} b_{1}\right) \\
& \leq \frac{3}{4} \mathrm{ulp}\left(a_{0} a_{1}\right) \\
& \leq \epsilon \cdot\left(\frac{3}{2} a_{0} a_{1}\right)
\end{aligned}
$$

and consequently

$$
\begin{aligned}
\left|z_{2}-z_{0} z_{1}\right| & =\sqrt{\Re\left(z_{2}-z_{0} z_{1}\right)^{2}+\Im\left(z_{2}-z_{0} z_{1}\right)^{2}} \\
& <\epsilon \sqrt{\left(\frac{3}{2} a_{0} a_{1}\right)^{2}+\left(2 a_{0} b_{1}+2 b_{0} a_{1}\right)^{2}} \\
& =\epsilon \sqrt{\frac{256}{55}\left|z_{0} z_{1}\right|^{2}-\frac{36}{55}\left(a_{0} b_{1}-b_{0} a_{1}\right)^{2}-\frac{1}{220}\left(23 a_{0} a_{1}-32 b_{0} b_{1}\right)^{2}} \\
& \leq \epsilon \sqrt{256 / 55}\left|z_{0} z_{1}\right|<\epsilon \sqrt{5}\left|z_{0} z_{1}\right|
\end{aligned}
$$

as required. 
Case R4. ulp $\left(a_{0} \otimes a_{1}-b_{0} \otimes b_{1}\right)<\operatorname{ulp}\left(b_{0} b_{1}\right)=u l p\left(a_{0} a_{1}\right)$.

In this case, there is again no rounding error introduced in computing the difference between $a_{0} \otimes a_{1}$ and $b_{0} \otimes b_{1}$, so we obtain

$$
\begin{aligned}
\left|\Re\left(z_{2}-z_{0} z_{1}\right)\right| & \leq\left|a_{0} \otimes a_{1}-a_{0} a_{1}\right|+\left|b_{0} \otimes b_{1}-b_{0} b_{1}\right| \\
& <\epsilon \cdot\left(a_{0} a_{1}+b_{0} b_{1}\right),
\end{aligned}
$$

and consequently,

$$
\begin{aligned}
\left|z_{2}-z_{0} z_{1}\right| & =\sqrt{\Re\left(z_{2}-z_{0} z_{1}\right)^{2}+\Im\left(z_{2}-z_{0} z_{1}\right)^{2}} \\
& <\epsilon \sqrt{\left(a_{0} a_{1}+b_{0} b_{1}\right)^{2}+\left(2 a_{0} b_{1}+2 b_{0} a_{1}\right)^{2}} \\
& =\epsilon \sqrt{5\left|z_{0} z_{1}\right|^{2}-\left(a_{0} b_{1}-b_{0} a_{1}\right)^{2}-4\left(a_{0} a_{1}-b_{0} b_{1}\right)^{2}} \\
& \leq \epsilon \sqrt{5}\left|z_{0} z_{1}\right|
\end{aligned}
$$

as required.

\section{WORST-CASE MULTIPLICANDS FOR $\beta=2$}

Having proved an upper bound on the relative error which can result from floating-point rounding when computing the product of complex values, we now turn to a more number-theoretic problem: finding precise worst-case inputs for $\beta=2$. Starting with the assumption that some inputs produce errors very close to the proven upper bound, we will repeatedly reduce the set of possible inputs until an exhaustive search becomes feasible.

Theorem 2. Let $\beta=2$ and assume that $z_{0}=a_{0}+b_{0} i \neq 0$ and $z_{1}=a_{1}+b_{1} i \neq 0$, where $a_{0}, b_{0}, a_{1}, b_{1}$ are floating-point values with $t$-digit base- $\beta$ significands, and $z_{2}=\left(\left(a_{0} \otimes a_{1}\right) \ominus\left(b_{0} \otimes b_{1}\right)\right)+\left(\left(a_{0} \otimes b_{1}\right) \oplus\left(b_{0} \otimes a_{1}\right)\right) i$ are such that

(11)

(2)

and no overflow, underflow, or denormal values occur during the computation of $z_{2}$. Assume further that the results of arithmetic operations are correctly rounded to a nearest representable value and that

$$
\frac{\left|z_{2}-z_{0} z_{1}\right|}{\left|z_{0} z_{1}\right|}>\epsilon \sqrt{5-n \epsilon}>\epsilon \cdot \max (\sqrt{1024 / 207}, \sqrt{32 / 7}+2 \epsilon)
$$

for some positive integer $n$. Then

$$
\begin{gathered}
a_{0} a_{1}=1 / 2+\left(j_{a a}+1 / 2\right) \epsilon+k_{a a} \epsilon^{2}, \\
a_{0} b_{1}=1 / 2+\left(j_{a b}+1 / 2\right) \epsilon+k_{a b} \epsilon^{2}, \\
b_{0} a_{1}=1 / 2+\left(j_{b a}+1 / 2\right) \epsilon+k_{b a} \epsilon^{2}, \\
b_{0} b_{1}=1 / 2+\left(j_{b b}+1 / 2\right) \epsilon+k_{b b} \epsilon^{2},
\end{gathered}
$$


for some integers $j_{x y}, k_{x y}$ satisfying

$$
\begin{gathered}
0 \leq j_{a a}, j_{a b}, j_{b a}, j_{b b}<\frac{n}{4}, \\
\left|k_{a a}\right|,\left|k_{b b}\right|<n, \\
\left|k_{a b}\right|,\left|k_{b a}\right|<\frac{n}{2}
\end{gathered}
$$

and $a_{0} \neq b_{0}, a_{1} \neq b_{1}$.

Proof. From equation (5), we note that $\epsilon \leq n \epsilon<11 / 207<2^{-4}$; we will use this trivial bound later without explicit comment.

From the proof of Theorem 1, we know that Case R4 must hold, i.e., there is no error introduced in the computation of the difference between $a_{0} \otimes a_{1}$ and $b_{0} \otimes b_{1}$, and $u l p\left(b_{0} b_{1}\right)=\mathrm{ulp}\left(a_{0} a_{1}\right)$. From inequalities (2) and (4) above, this implies that

$$
\begin{gathered}
1 / 2 \leq b_{0} b_{1} \leq a_{0} a_{1}<1, \\
\left|\Re\left(z_{2}-z_{0} z_{1}\right)\right| \leq\left|a_{0} \otimes a_{1}-a_{0} a_{1}\right|+\left|b_{0} \otimes b_{1}-b_{0} b_{1}\right| \leq \epsilon .
\end{gathered}
$$

We can now obtain lower bounds on $\left|z_{0} z_{1}\right|$ and $\left|z_{2}-z_{0} z_{1}\right|$, using the fact that $\left(a_{0} a_{1}\right)\left(b_{0} b_{1}\right)=\left(a_{0} b_{1}\right)\left(b_{0} a_{1}\right)$ :

$$
\begin{aligned}
\left|z_{0} z_{1}\right|^{2} & =\left(a_{0}^{2}+b_{0}^{2}\right)\left(a_{1}^{2}+b_{1}^{2}\right) \\
& =\left(a_{0} a_{1}\right)^{2}+\left(a_{0} b_{1}\right)^{2}+\left(b_{0} a_{1}\right)^{2}+\left(b_{0} b_{1}\right)^{2} \\
& \geq(1 / 2)^{2}+\left(a_{0} b_{1}\right)^{2}+\frac{(1 / 2)^{4}}{\left(a_{0} b_{1}\right)^{2}}+(1 / 2)^{2} \geq 1, \\
\left|z_{2}-z_{0} z_{1}\right|^{2} & >\left|z_{0} z_{1}\right|^{2} \epsilon^{2}(5-n \epsilon) \geq \epsilon^{2}(5-n \epsilon),
\end{aligned}
$$

as well as an upper bound on $\left|z_{0} z_{1}\right|$ :

$$
\begin{aligned}
\left|z_{0} z_{1}\right|^{2} \cdot \frac{1024 \epsilon^{2}}{207} & <\left|z_{2}-z_{0} z_{1}\right|^{2} \\
& =\left|\Re\left(z_{2}-z_{0} z_{1}\right)\right|^{2}+\left|\Im\left(z_{2}-z_{0} z_{1}\right)\right|^{2} \\
& <\epsilon^{2}+\left(\epsilon \cdot\left(2 a_{0} b_{1}+2 b_{0} a_{1}\right)\right)^{2} \\
& \leq \epsilon^{2}+4 \epsilon^{2}\left|z_{0} z_{1}\right|^{2} \\
\left|z_{0} z_{1}\right|^{2} & <\frac{207}{196}
\end{aligned}
$$

We now note that

$$
\begin{aligned}
\left(a_{0} b_{1}\right)^{2} & \leq\left|z_{0} z_{1}\right|^{2}-\left(a_{0} a_{1}\right)^{2}-\left(b_{0} b_{1}\right)^{2} \\
& \leq \frac{207}{196}-\frac{1}{4}-\frac{1}{4}=\frac{109}{196},
\end{aligned}
$$

so $b_{0} a_{1} \leq a_{0} b_{1} \leq \sqrt{109 / 196}<1$ and $a_{0} \otimes b_{1}+b_{0} \otimes a_{1} \leq \sqrt{109 / 49} \cdot(1+\epsilon)<2$; this implies that $\operatorname{ulp}\left(b_{0} a_{1}\right) \leq \mathrm{ulp}\left(a_{0} b_{1}\right) \leq \mathrm{ulp}(1 / 2)$ and $\mathrm{ulp}\left(a_{0} \otimes b_{1}+b_{0} \otimes a_{1}\right) \leq \mathrm{ulp}(1)$, and therefore

$$
\begin{gathered}
\left|a_{0} \otimes b_{1}-a_{0} b_{1}\right| \leq \epsilon / 2, \\
\left|b_{0} \otimes a_{1}-b_{0} a_{1}\right| \leq \epsilon / 2, \\
\left|\left(\left(a_{0} \otimes b_{1}\right) \oplus\left(b_{0} \otimes a_{1}\right)\right)-\left(a_{0} \otimes b_{1}+b_{0} \otimes a_{1}\right)\right| \leq \epsilon, \\
\left|\Im\left(z_{2}-z_{0} z_{1}\right)\right| \leq \epsilon / 2+\epsilon / 2+\epsilon=2 \epsilon,
\end{gathered}
$$


which allows us to place upper bounds on $\left|z_{2}-z_{0} z_{1}\right|$ and $\left|z_{0} z_{1}\right|$ :

$$
\begin{gathered}
\left|z_{2}-z_{0} z_{1}\right|^{2}=\left|\Re\left(z_{2}-z_{0} z_{1}\right)\right|^{2}+\left|\Im\left(z_{2}-z_{0} z_{1}\right)\right|^{2} \leq(\epsilon)^{2}+(2 \epsilon)^{2}=5 \epsilon^{2}, \\
\left|z_{0} z_{1}\right|^{2}<\frac{\left|z_{2}-z_{0} z_{1}\right|^{2}}{\epsilon^{2}(5-n \epsilon)} \leq \frac{5}{5-n \epsilon} .
\end{gathered}
$$

Combining the known lower bound $\epsilon^{2}(5-n \epsilon)$ for $\left|z_{2}-z_{0} z_{1}\right|^{2}$ with the upper bounds on the error contributed by each individual rounding step, we find that

$$
\begin{gathered}
\epsilon / 2-(1-\sqrt{1-n \epsilon}) \epsilon<\left|a_{0} \otimes a_{1}-a_{0} a_{1}\right| \leq \epsilon / 2, \\
\epsilon / 2-(1-\sqrt{1-n \epsilon}) \epsilon<\left|b_{0} \otimes b_{1}-b_{0} b_{1}\right| \leq \epsilon / 2, \\
\epsilon / 2-(2-\sqrt{4-n \epsilon}) \epsilon<\left|a_{0} \otimes b_{1}-a_{0} b_{1}\right| \leq \epsilon / 2, \\
\epsilon / 2-(2-\sqrt{4-n \epsilon}) \epsilon<\left|b_{0} \otimes a_{1}-b_{0} a_{1}\right| \leq \epsilon / 2,
\end{gathered}
$$

and similarly, by combining the upper bound on $\left|z_{0} z_{1}\right|^{2}$ with the lower bound of $1 / 2$ for each pairwise product, we obtain

$$
\begin{aligned}
& 1 / 2 \leq b_{0} b_{1} \leq a_{0} a_{1} \leq \sqrt{\frac{5}{5-n \epsilon}-\frac{3}{4}}=\sqrt{\frac{5+3 n \epsilon}{20-4 n \epsilon}} \\
& 1 / 2 \leq b_{0} a_{1} \leq a_{0} b_{1} \leq \sqrt{\frac{5}{5-n \epsilon}-\frac{3}{4}}=\sqrt{\frac{5+3 n \epsilon}{20-4 n \epsilon}}
\end{aligned}
$$

Now consider the possible values for $a_{0} a_{1}$ which satisfy these restrictions. Since it is the product of two values which are expressible using $t$ digits of significand, $a_{0} a_{1}$ can be exactly represented using $2 t$ digits of significand; but since $1 / 2 \leq a_{0} a_{1}<1$, this implies that $a_{0} a_{1}$ is an integer multiple of $\epsilon^{2}$. Therefore there is at least one pair of integers $j_{a a}, k_{a a}$ with $0 \leq j_{a a}<\epsilon^{-1} / 2,\left|k_{a a}\right| \leq \epsilon^{-1} / 2$ for which

$$
a_{0} a_{1}=1 / 2+\left(j_{a a}+1 / 2\right) \epsilon+k_{a a} \epsilon^{2} .
$$

Since $a_{0} \otimes a_{1}$ is the closest multiple of $\epsilon$ to $a_{0} a_{1}$, this implies that

$$
\begin{gathered}
\epsilon / 2-(1-\sqrt{1-n \epsilon}) \epsilon<\left|a_{0} \otimes a_{1}-a_{0} a_{1}\right|=\epsilon / 2-\left|k_{a a}\right| \epsilon^{2}, \\
\left|k_{a a}\right| \epsilon<1-\sqrt{1-n \epsilon}<1-(1-n \epsilon)=n \epsilon,
\end{gathered}
$$

i.e., $\left|k_{a a}\right|<n$, and similarly,

$$
1 / 2+j_{a a} \epsilon \leq a_{0} a_{1} \leq \sqrt{\frac{5+3 n \epsilon}{20-4 n \epsilon}}<\sqrt{1 / 4+n \epsilon / 4}<1 / 2+\frac{n \epsilon}{4},
$$

i.e., $0 \leq j_{a a}<n / 4$.

Applying the same argument to $a_{0} b_{1}, b_{0} a_{1}$, and $b_{0} b_{1}$ allows us to infer that they possess the same structure, as required. To complete the proof, we note that the rounding errors from the products $a_{0} a_{1}$ and $b_{0} b_{1}$ must be in opposite directions (so that they accumulate when subtracted), while the rounding errors from the products $a_{0} b_{1}$ and $b_{0} a_{1}$ must be in the same direction (so that they accumulate when added); consequently, we must have $a_{0} \neq b_{0}$ and $a_{1} \neq b_{1}$. 
Corollary 1. Assume that the preconditions of Theorem 2 are satisfied, and assume further that

$$
\frac{1}{2} \leq a_{0}<1
$$

and $n \leq 2 \epsilon^{-1 / 2}$. Then

$$
\frac{1}{2}<a_{0}, b_{0}, a_{1}, b_{1}<1 .
$$

Proof. Assume that $a_{1} \geq 1$. Then we can write

$$
\begin{gathered}
a_{0}=1 / 2+A \epsilon, \\
a_{1}=1+2 B \epsilon,
\end{gathered}
$$

for some $0 \leq A, B<(2 \epsilon)^{-1}$. From Theorem 2, we have

$$
1 / 2+(A+B) \epsilon+2 A B \epsilon^{2}=a_{0} a_{1}=1 / 2+\left(j_{a a}+1 / 2\right) \epsilon+k_{a a} \epsilon^{2}
$$

for some $0 \leq j_{a a}<n / 4,\left|k_{a a}\right|<n$.

As a result, we must have $A+B \leq n / 4 \leq 1 / 2 \cdot \epsilon^{-1 / 2}$, and since $0 \leq A, B$, this implies $0 \leq 2 A B \epsilon^{2} \leq \epsilon / 8$. However, by reducing the equation above modulo $\epsilon$, we find that $2 A B \epsilon^{2} \equiv \epsilon / 2+k_{a a} \epsilon^{2}$, which contradicts our bounds on $2 A B \epsilon^{2}$. Consequently, we can conclude that $a_{1}<1$. Now we note that $a_{0} a_{1}>1 / 2$ and $a_{0}<1$, so $a_{1}>1 / 2$, and we have both of the bounds required for $a_{1}$.

Applying the same argument to the other products provides the same bounds for $a_{0}, b_{0}$, and $b_{1}$.

Corollary 2. Assume that the preconditions of Corollary 1 are satisfied, and assume further that $n \leq \epsilon^{-1 / 2}$ and $\epsilon \leq 2^{-6}$. Then

$$
\begin{gathered}
j_{a a}-j_{a b}-j_{b a}+j_{b b}=0, \\
\left|a_{0}-b_{0}\right| \cdot\left|a_{1}-b_{1}\right|<3 n \epsilon^{2} .
\end{gathered}
$$

Proof. From Theorem 2, we obtain that

$$
a_{0}\left(a_{1}-b_{1}\right)=a_{0} a_{1}-a_{0} b_{1}=\left(j_{a a}-j_{a b}\right) \epsilon+\left(k_{a a}-k_{a b}\right) \epsilon^{2}
$$

where $\left|j_{a a}-j_{a b}\right|<\frac{n}{4},\left|k_{a a}-k_{a b}\right|<\frac{3 n}{2}$, and since $a_{0}>\frac{1}{2}$ (from Corollary 11), we can conclude that $\left|a_{1}-b_{1}\right|<\frac{n}{2} \epsilon+3 n \epsilon^{2}$. Since $a_{1}$ and $b_{1}$ are integer multiples of $\epsilon$ and $3 n \epsilon^{2}<\epsilon / 2$, we conclude that $\left|a_{1}-b_{1}\right| \leq \frac{n}{2} \epsilon$. Applying the same argument to the product $a_{1}\left(a_{0}-b_{0}\right)$ provides the same bound for $\left|a_{0}-b_{0}\right|$.

We now note that

$$
\begin{aligned}
\left|\left(j_{a a}-j_{a b}-j_{b a}+j_{b b}\right) \epsilon+\left(k_{a a}-k_{a b}-k_{b a}+k_{b b}\right) \epsilon^{2}\right| & =\left|a_{0}-b_{0}\right| \cdot\left|a_{1}-b_{1}\right| \\
& \leq\left(\frac{n}{2} \epsilon\right)^{2} \\
& <\frac{\epsilon}{4}
\end{aligned}
$$

from our assumed upper bound on $n$, and consequently we can conclude that $j_{a a}-j_{a b}-j_{b a}+j_{b b}=0$. Finally, this allows us to write

$$
\begin{aligned}
\left|a_{0}-b_{0}\right| \cdot\left|a_{1}-b_{1}\right| & =\left|k_{a a}-k_{a b}-k_{b a}+k_{b b}\right| \epsilon^{2} \\
& <3 n \epsilon^{2}
\end{aligned}
$$

as required. 
Corollary 3. Assume that the preconditions of Corollary 1 are satisfied, and assume further that $n \leq \frac{1}{4} \epsilon^{-1 / 2}$. Then

$$
\begin{gathered}
\left(a_{0}-b_{0}\right)\left(a_{1}-b_{1}\right)=2\left(j_{a a}-j_{a b}\right)\left(j_{a a}-j_{b a}\right) \epsilon^{2}, \\
\left(a_{0}-b_{0}\right)\left(a_{1}-b_{1}\right) k_{a a}=\left(k_{a a}-k_{a b}\right)\left(k_{a a}-k_{b a}\right) \epsilon^{2} .
\end{gathered}
$$

Proof. For brevity and clarity, we will write $\left(a_{0}-b_{0}\right)\left(a_{1}-b_{1}\right)=x \epsilon^{2}$ and note that $x$ is an integer between $-3 n$ and $3 n$, from Corollary 2 , Then

$$
\begin{aligned}
x a_{0} a_{1}= & \frac{x}{2}+x\left(j_{a a}+\frac{1}{2}\right) \epsilon+x k_{a a} \epsilon^{2}, \\
x a_{0} a_{1}= & \frac{a_{0}\left(a_{1}-b_{1}\right)}{\epsilon} \cdot \frac{a_{1}\left(a_{0}-b_{0}\right)}{\epsilon} \\
= & \left(\left(j_{a a}-j_{a b}\right)+\left(k_{a a}-k_{a b}\right) \epsilon\right)\left(\left(j_{a a}-j_{b a}\right)+\left(k_{a a}-k_{b a}\right) \epsilon\right) \\
= & \left(j_{a a}-j_{a b}\right)\left(j_{a a}-j_{b a}\right)+\left(\left(j_{a a}-j_{a b}\right)\left(k_{a a}-k_{b a}\right)+\left(j_{a a}-j_{b a}\right)\left(k_{a a}-k_{a b}\right)\right) \epsilon \\
& \quad+\left(k_{a a}-k_{a b}\right)\left(k_{a a}-k_{b a}\right) \epsilon^{2} .
\end{aligned}
$$

Consequently,

$$
\begin{aligned}
x-2\left(j_{a a}-\right. & \left.j_{a b}\right)\left(j_{a a}-j_{b a}\right) \\
= & \left(2\left(j_{a a}-j_{a b}\right)\left(k_{a a}-k_{b a}\right)\right. \\
& \left.+2\left(j_{a a}-j_{b a}\right)\left(k_{a a}-k_{a b}\right)-\left(2 j_{a a}+1\right) x\right) \epsilon \\
& +\left(2\left(k_{a a}-k_{a b}\right)\left(k_{a a}-k_{b a}\right)-2 k_{a a} x\right) \epsilon^{2}, \\
\left|x-2\left(j_{a a}-j_{a b}\right)\left(j_{a a}-j_{b a}\right)\right| \leq & \left(2 \frac{n}{4} \frac{3 n}{2}+2 \frac{n}{4} \frac{3 n}{2}+3\left(\frac{n}{2}+1\right) n\right) \epsilon \\
& +\left(2 \frac{3 n}{2} \frac{3 n}{2}+6 n^{2}\right) \epsilon^{2} \\
= & \left(3 n^{2}+3 n\right) \epsilon+\frac{21}{2} n^{2} \epsilon^{2} \\
\leq & \frac{3}{16}+\frac{3}{4} \sqrt{\epsilon}+\frac{21}{32} \epsilon<1,
\end{aligned}
$$

and since the only integer with absolute value less than one is zero, we can conclude that $x=2\left(j_{a a}-j_{a b}\right)\left(j_{a a}-j_{b a}\right)$ as required.

We now consider $x a_{0} a_{1} \epsilon^{-2}$ modulo $\frac{1}{2} \epsilon^{-1}$, and note that

$$
\begin{aligned}
x k_{a a} & \equiv x a_{0} a_{1} \epsilon^{-2} \\
& \equiv\left(k_{a a}-k_{a b}\right)\left(k_{a a}-k_{b a}\right)
\end{aligned}
$$

and further that

$$
\begin{aligned}
\left|x k_{a a}-\left(k_{a a}-k_{a b}\right)\left(k_{a a}-k_{b a}\right)\right| & \leq 3 n \cdot n+\frac{3 n}{2} \cdot \frac{3 n}{2} \\
& =\frac{21 n^{2}}{4} \leq \frac{21 \epsilon^{-1}}{64}<\frac{1}{2} \epsilon^{-1}
\end{aligned}
$$

and therefore $x k_{a a}=\left(k_{a a}-k_{a b}\right)\left(k_{a a}-k_{b a}\right)$. 
Theorem 3. Let $\beta=2$ and assume that $z_{0}=a_{0}+b_{0} i, z_{1}=a_{1}+b_{1} i$, and $z_{2}=\left(\left(a_{0} \otimes a_{1}\right) \ominus\left(b_{0} \otimes b_{1}\right)\right)+\left(\left(a_{0} \otimes b_{1}\right) \oplus\left(b_{0} \otimes a_{1}\right)\right) i$ are such that

(11)

(21)

(3)

(4)

(6)

$$
\begin{aligned}
0 & \leq a_{0}, b_{0}, a_{1}, b_{1}, \\
b_{0} b_{1} & \leq a_{0} a_{1}, \\
b_{0} a_{1} & \leq a_{0} b_{1}, \\
1 / 2 & \leq a_{0} a_{1}<1, \\
1 / 2 & \leq a_{0}<1,
\end{aligned}
$$

and no overflow, underflow, or denormal values occur during the computation of $z_{2}$. Assume further that the results of arithmetic operations are correctly rounded to a nearest representable value, and that

$$
\frac{\left|z_{2}-z_{0} z_{1}\right|}{\left|z_{0} z_{1}\right|}>\epsilon \sqrt{5-n \epsilon}>\epsilon \cdot \max (\sqrt{1024 / 207}, \sqrt{32 / 7}+2 \epsilon)
$$

for some $n<\frac{1}{4} \epsilon^{-1 / 2}$ and $\epsilon \leq 2^{-6}$. Then there exist integers $c_{0}, d_{0}, \alpha_{0}, \beta_{0}, c_{1}, d_{1}$, $\alpha_{1}, \beta_{1}$ satisfying

$$
\begin{array}{cr}
a_{0}=\frac{c_{0}}{d_{0}}\left(1+\alpha_{0} \epsilon\right) & b_{0}=\frac{c_{0}}{d_{0}}\left(1+\beta_{0} \epsilon\right) \\
a_{1}=\frac{c_{1}}{d_{1}}\left(1+\alpha_{1} \epsilon\right) & b_{1}=\frac{c_{1}}{d_{1}}\left(1+\beta_{1} \epsilon\right) \\
\operatorname{gcd}\left(c_{0}, d_{0}\right)=1 & \frac{d_{0}}{2} \leq c_{0} \leq d_{0} \\
\operatorname{gcd}\left(c_{1}, d_{1}\right)=1 & \frac{d_{1}}{2} \leq c_{1} \leq d_{1} \\
2 c_{0} c_{1}=d_{0} d_{1}<3 n & \frac{1}{2}<a_{0}, b_{0}, a_{1}, b_{1}<1 \\
\alpha_{0} \equiv \beta_{0} \equiv-\epsilon^{-1} \quad\left(\bmod d_{0}\right) & \alpha_{0} \neq \beta_{0} \\
\alpha_{1} \equiv \beta_{1} \equiv-\epsilon^{-1} \quad\left(\bmod d_{1}\right) & \alpha_{1} \neq \beta_{1} \\
\min \left(\alpha_{0}, \beta_{0}\right)+\min \left(\alpha_{1}, \beta_{1}\right) \geq 0 & \max \left(\left|\alpha_{0}\right|,\left|\beta_{0}\right|\right) \cdot \max \left(\left|\alpha_{1}\right|,\left|\beta_{1}\right|\right)<n .
\end{array}
$$

Proof. Let the values $j_{a a}, j_{a b}, j_{b a}, j_{b b}, k_{a a}, k_{a b}, k_{b a}$, and $k_{b b}$ be as constructed in Theorem 2] and further let $g_{0}=\operatorname{gcd}\left(j_{a a}-j_{a b},\left(a_{1}-b_{1}\right) / \epsilon\right)$. From Corollary 1 we know that $1 / 2<a_{1}, b_{1}<1$, so $a_{1}$ and $b_{1}$ are multiples of $\epsilon$; consequently, $g_{0}$ must be an integer. By the same argument, $g_{1}=\operatorname{gcd}\left(j_{a a}-j_{b a},\left(a_{0}-b_{0}\right) / \epsilon\right)$ is an integer.

Now note that

$$
g_{0}\left|\left(a_{1}-b_{1}\right) \epsilon^{-1}\right|\left(a_{1}-b_{1}\right) a_{0} \epsilon^{-2}=\left(j_{a a}-j_{a b}\right) \epsilon^{-1}+\left(k_{a a}-k_{a b}\right)
$$

and since $g_{0} \mid\left(j_{a a}-j_{a b}\right)$, we can conclude that $g_{0} \mid\left(k_{a a}-k_{a b}\right)$. By the same argument, $g_{1} \mid\left(k_{a a}-k_{b a}\right)$.

We now write

$$
\begin{array}{lll}
c_{0}=\frac{j_{a a}-j_{a b}}{g_{0}} & d_{0}=\frac{a_{1}-b_{1}}{g_{0} \epsilon} & e_{0}=\frac{k_{a a}-k_{a b}}{g_{0}} \\
c_{1}=\frac{j_{a a}-j_{b a}}{g_{1}} & d_{1}=\frac{a_{0}-b_{0}}{g_{1} \epsilon} & e_{1}=\frac{k_{a a}-k_{b a}}{g_{1}}
\end{array}
$$

and note that these values are all integers; further, from Corollary 3 we have $d_{0} d_{1} k_{a a}=e_{0} e_{1}$ and $d_{0} d_{1}=2 c_{0} c_{1}$, and since $\operatorname{gcd}\left(c_{0}, d_{0}\right)=\operatorname{gcd}\left(c_{1}, d_{1}\right)=1$ by construction, this implies $\operatorname{gcd}\left(c_{0}, c_{1}\right)=1$. 
We now observe that

$$
\begin{aligned}
& a_{0}=\frac{a_{0}\left(a_{1}-b_{1}\right)}{a_{1}-b_{1}}=\frac{c_{0} g_{0} \epsilon+e_{0} g_{0} \epsilon^{2}}{d_{0} g_{0} \epsilon}=\frac{c_{0}+e_{0} \epsilon}{d_{0}}, \\
& a_{1}=\frac{a_{1}\left(a_{0}-b_{0}\right)}{a_{0}-b_{0}}=\frac{c_{1} g_{1} \epsilon+e_{1} g_{1} \epsilon^{2}}{d_{1} g_{1} \epsilon}=\frac{c_{1}+e_{1} \epsilon}{d_{1}},
\end{aligned}
$$

and therefore

$$
\begin{aligned}
\frac{1}{2}+ & \left(j_{a a}+\frac{1}{2}\right) \epsilon+k_{a a} \epsilon^{2}=a_{0} a_{1} \\
& =\frac{c_{0} c_{1}}{d_{0} d_{1}}+\frac{c_{0} e_{1}+e_{0} c_{1}}{d_{0} d_{1}} \epsilon+\frac{e_{0} e_{1}}{d_{0} d_{1}} \epsilon^{2} \\
& =\frac{1}{2}+\frac{c_{0} e_{1}+e_{0} c_{1}}{d_{0} d_{1}} \epsilon+k_{a a} \epsilon^{2},
\end{aligned}
$$

and thus (using $d_{0} d_{1}=2 c_{0} c_{1}$ ),

$$
c_{0} c_{1}\left(2 j_{a a}+1\right)=c_{0} e_{1}+e_{0} c_{1} .
$$

Consequently, $c_{0} \mid e_{0} c_{1}$ and $c_{1} \mid c_{0} e_{1}$, and since $\operatorname{gcd}\left(c_{0}, c_{1}\right)=1$, it follows that $c_{0} \mid e_{0}$ and $c_{1} \mid e_{1}$. Writing $e_{0}=c_{0} \alpha_{0}, e_{1}=c_{1} \alpha_{1}$ for integers $\alpha_{0}, \alpha_{1}$, we now have

$$
a_{0}=\frac{c_{0}}{d_{0}}\left(1+\alpha_{0} \epsilon\right) \quad a_{1}=\frac{c_{1}}{d_{1}}\left(1+\alpha_{1} \epsilon\right)
$$

and taking $\beta_{0}=\alpha_{0}+2 c_{1} g_{1}, \beta_{1}=\alpha_{1}+2 c_{0} g_{0}$, we have

$$
b_{0}=\frac{c_{0}}{d_{0}}\left(1+\beta_{0} \epsilon\right) \quad b_{1}=\frac{c_{1}}{d_{1}}\left(1+\beta_{1} \epsilon\right)
$$

as required.

The remaining conditions can be obtained by remembering that $a_{0}, b_{0}, a_{1}$, and $b_{1}$ are integer multiples of $\epsilon$, and by using the bounds on $j_{x y}$ and $k_{x y}$ given in Theorem 2

Corollary 4. In IEEE 754 single-precision arithmetic $\left(\beta=2, t=24, \epsilon=2^{-24}\right)$, using "nearest even" rounding mode, the value@ 1

$$
a_{0}=\frac{3}{4} \quad b_{0}=\frac{3}{4}(1-4 \epsilon) \quad a_{1}=\frac{2}{3}(1+11 \epsilon) \quad b_{1}=\frac{2}{3}(1+5 \epsilon)
$$

result in a relative error $\delta \approx \epsilon \sqrt{5-168 \epsilon} \approx \epsilon \sqrt{4.9999899864}$ in $z_{2}$, and $\delta$ is the largest possible relative error for IEEE 754 single-precision inputs provided that overflow, underflow, and denormals do not occur.

\footnotetext{
${ }^{1}$ Note that while $\frac{2}{3}$ is not an IEEE 754 single-precision value, $\frac{2}{3}(1+5 \epsilon)$ and $\frac{2}{3}(1+11 \epsilon)$ are, since $\epsilon^{-1}+5 \equiv \epsilon^{-1}+11 \equiv 0(\bmod 3)$.
} 
Proof. Straightforward computation for the values given establishes that

$$
\begin{array}{rlrl}
a_{0} a_{1} & =\frac{1}{2}(1+11 \epsilon) & a_{0} \otimes a_{1} & =\frac{1}{2}(1+12 \epsilon) \\
b_{0} b_{1} & =\frac{1}{2}\left(1+\epsilon-20 \epsilon^{2}\right) & b_{0} \otimes b_{1} & =\frac{1}{2} \\
\Re\left(z_{0} z_{1}\right) & =5 \epsilon+10 \epsilon^{2} & \Re\left(z_{2}\right) & =6 \epsilon \\
a_{0} b_{1} & =\frac{1}{2}(1+5 \epsilon) & a_{0} \otimes b_{1} & =\frac{1}{2}(1+4 \epsilon) \\
b_{0} a_{1} & =\frac{1}{2}\left(1+7 \epsilon-44 \epsilon^{2}\right) & b_{0} \otimes a_{1} & =\frac{1}{2}(1+6 \epsilon) \\
\Im\left(z_{0} z_{1}\right) & =1+6 \epsilon-22 \epsilon^{2} & \Im\left(z_{2}\right) & =1+4 \epsilon \\
\left|z_{2}-z_{0} z_{1}\right|^{2} & =\epsilon^{2}\left(5-108 \epsilon+\mathrm{O}\left(\epsilon^{2}\right)\right) \\
\left|z_{0} z_{1}\right|^{2} & =1+12 \epsilon+\mathrm{O}\left(\epsilon^{2}\right) &
\end{array}
$$

and the ratio of these provides the error as stated.

To prove that this is the largest possible relative error for IEEE single-precision inputs, we note that the mappings $z_{0} \rightarrow z_{0} i, z_{1} \rightarrow z_{1} i,\left(z_{0}, z_{1}\right) \rightarrow\left(\overline{z_{0}}, \overline{z_{1}}\right),\left(z_{0}, z_{1}\right) \rightarrow$ $\left(z_{1}, z_{0}\right), z_{0} \rightarrow z_{0} \cdot 2^{j}$, and $z_{1} \rightarrow z_{1} \cdot 2^{k}$ do not affect the relative error in $z_{2}$; consequently, this allows us to assume, without loss of generality, that conditions (11) -(4) and (6) are satisfied by the worst-case inputs. Using the results of Theorem 3. an exhaustive computer search (taking about five minutes in MAPLE on the second author's $1.4 \mathrm{GHz}$ laptop) completes the proof.

Corollary 5. In IEEE 754 double-precision arithmetic $\left(\beta=2, t=53, \epsilon=2^{-53}\right)$, using "nearest even" rounding mode, the values

$$
a_{0}=\frac{3}{4}(1+4 \epsilon) \quad b_{0}=\frac{3}{4} \quad a_{1}=\frac{2}{3}(1+7 \epsilon) \quad b_{1}=\frac{2}{3}(1+\epsilon)
$$

result in a relative error in $z_{2}$ of approximately $\epsilon \sqrt{5-96 \epsilon} \approx \epsilon \sqrt{4.9999999999999893}$, and this is the worst possible provided that overflow, underflow, and denormals do not occur.

Proof. Straightforward computation for the values given establishes that

$$
\begin{aligned}
\left|z_{2}-z_{0} z_{1}\right|^{2} & =\epsilon^{2}\left(5-36 \epsilon+\mathrm{O}\left(\epsilon^{2}\right)\right), \\
\left|z_{0} z_{1}\right|^{2} & =1+12 \epsilon+\mathrm{O}\left(\epsilon^{2}\right)
\end{aligned}
$$

and the ratio of these provides the error as stated.

As in Corollary 4, an exhaustive search using the results of Theorem 3 (again, taking just a few minutes) completes the proof.

For $\beta=2$ and $t>6$, the constructions given in Corollaries 4 and 5 for $a_{0}, b_{0}, a_{1}$, $b_{1}$ provide for even and odd $t$, respectively, relative errors of $\epsilon \sqrt{5-168 \epsilon+\mathrm{O}\left(\epsilon^{2}\right)}$ and $\epsilon \sqrt{5-96 \epsilon+\mathrm{O}\left(\epsilon^{2}\right)}$. We believe that these are the worst-case inputs for all sufficiently large $t$ when $\beta=2$; details will be given in a forthcoming Technical Report. 


\section{A Note On MEthods}

The existence of this paper serves as a strong demonstration of the power of experimental mathematics. The initial result - the upper bound of $\sqrt{5} \epsilon$ - was discovered experimentally seven years ago, on the basis of testing a few million random single-precision products.

Experimental methods became even more important when it came to the results concerning worst-case inputs. Here the approach taken was to perform an exhaustive search, taking several hours on the second author's laptop, of IEEE single-precision inputs, using only a few arguments from Theorem 1 to prune the search. Once the worst few sets of inputs had been enumerated, it became clear that they possessed the structure described in Theorem 3, and it was natural to conjecture that this structure would be satisfied by the worst-case inputs in any precision. As is common with such problems, once the required result was known, constructing a proof was fairly straightforward.

\section{REFERENCES}

1. N.J. Higham, Accuracy and Stability of Numerical Algorithms, Second Edition, SIAM, 2002. MR.1927606 (2003g:65064)

2. C. Percival, Rapid multiplication modulo the sum and difference of highly composite numbers, Math. Comp. 72 (2002), 387-395. MR.1933827 (2003i:11183)

Mathematical Sciences Institute, Australian National University, Canberra, ACt 0200, Australia

E-mail address: complex@rpbrent.com

iRmaCs Centre, Simon Fraser University, Burnaby, BC, Canada

E-mail address: cperciva@irmacs.sfu.ca

INRIA LoRraine/LORIA, 615 RUE DU JARdin Botanique, F-54602 Villers-Lès-NANCY Cedex, France

E-mail address: zimmerma@loria.fr 